\title{
Metastability of Stochastic Partial Differential Equations and Fredholm Determinants
}

\author{
Nils Berglund (Université d'Orléans, France)
}

\begin{abstract}
Metastability occurs when a thermodynamic system, such as supercooled water (which is liquid even below freezing point), lands on the "wrong" side of a phase transition, and remains in a state which differs from its equilibrium state for a considerable time. There are numerous mathematical models describing this phenomenon, including lattice models with stochastic dynamics. In this text, we will be interested in metastability in parabolic stochastic partial differential equations (SPDEs). Some of these equations are ill posed, and only thanks to very recent progress in the theory of so-called singular SPDEs does one know how to construct solutions via a renormalisation procedure. The study of metastability in these systems reveals unexpected links with the theory of spectral determinants, including Fredholm and Carleman-Fredholm determinants.
\end{abstract}

\section{Introduction}

Put a water bottle in your freezer. If the water is pure enough, when you take it out after a few hours you will find that the water is still in its liquid state although at a negative temperature. One says that the water is supercooled. Shake the bottle and you will see the water suddenly turn into ice.

Supercooled water is an example of a metastable state. In such a state, a thermodynamic potential of a physical system, such as its free energy, is minimised locally but not globally. The transition to its stable state requires the system to jump over an energy barrier, and this may take a long time if only fluctuations due to thermal agitation play a role. Thus, the transformation of supercooled water into ice is achieved by nucleation, that is, by the appearance of ice crystals that grow slowly, ${ }^{1}$ although the presence of impurities or an outside energy source can speed up the solidification process.

There are many mathematical models describing metastability phenomena. The first to have been studied were lattice models such as the Ising model with a Metropolis-Hastings type stochastic dynamics. See for instance [8] for an overview of metastability results in lattice systems. Metastability also occurs in continuous systems such as stochastic (ordinary)

\footnotetext{
1 A spherical crystal with radius $r$ changes the system's energy in two ways: since the ice is more stable than the liquid water, the energy is decreased by an amount proportional to the crystal volume, $r^{3}$; however the interface between the crystal and the surrounding water increases the energy by a quantity proportional to the crystal's surface, $r^{2}$. For small values of $r$, the second contribution dominates the first one, whereas the opposite holds for large $r$. Therefore, the ice crystals grow rather slowly as long as their size is smaller than a critical value, for which the volume and the surface terms are comparable.
}

differential equations, which we will consider in Section 2, and in stochastic partial differential equations that will be addressed in Section 3.

\section{Reversible diffusions}

The motion in $\mathbb{R}^{n}$ of a Brownian particle of mass $m$, subjected to a force arising from a potential $V$, a viscous damping force and thermal fluctuations can be described by Langevin's equation

$$
m \frac{\mathrm{d}^{2} x_{t}}{\mathrm{~d} t^{2}}=-\nabla V\left(x_{t}\right)-\gamma \frac{\mathrm{d} x_{t}}{\mathrm{~d} t}+\sigma \frac{\mathrm{d} W_{t}}{\mathrm{~d} t},
$$

where $W_{t}$ is a Brownian motion (see Appendix A), $\gamma$ is a damping coefficient and the positive parameter $\sigma$ is related to the temperature. We will assume in what follows that $V: \mathbb{R}^{n} \rightarrow \mathbb{R}$ is a confining potential (bounded below and converging to infinity quickly enough), and we are mainly interested in the case of small $\sigma$. In order to simplify a number of expressions we will write $\sigma=\sqrt{2 \varepsilon}$.

When $\varepsilon=0$, if the mass $m$ is small compared with the damping coefficient $\gamma$, the particle converges without oscillating to a local minimium of $V$. In that case, the motion is said to be overdamped. For any $\varepsilon$ and in the limit of very small $m / \gamma$, one can show that after a change of variables the dynamics is described by the simpler first-order equation

$$
\frac{\mathrm{d} x_{t}}{\mathrm{~d} t}=-\nabla V\left(x_{t}\right)+\sqrt{2 \varepsilon} \frac{\mathrm{d} W_{t}}{\mathrm{~d} t},
$$

called an overdamped Langevin equation. Mathematically speaking, this is an example of a stochastic differential equation (SDE) and its solution is also called a diffusion.

For instance, in dimension $n=1$, if $V(x)=\frac{1}{2} x^{2}$ equation (1) becomes

$$
\frac{\mathrm{d} x_{t}}{\mathrm{~d} t}=-x_{t}+\sqrt{2 \varepsilon} \frac{\mathrm{d} W_{t}}{\mathrm{~d} t},
$$

and describes an overdamped harmonic oscillator subject to thermal noise. Its solution is called an Ornstein-Uhlenbeck process.

One way to describe the solutions of (1) is to determine their transition probabilities $p_{t}(x, y)$. These are such that if a particle starts from the point $x$ at time 0 , then the probability $\mathbb{P}^{x}\left\{x_{t} \in A\right\}$ of finding it in a region $A$ at time $t>0$ is given by

$$
\mathbb{P}^{x}\left\{x_{t} \in A\right\}=\int_{A} p_{t}(x, y) \mathrm{d} y .
$$

It is known that $p_{t}(x, y)$ satisfies the Fokker-Planck equation

$$
\partial_{t} p_{t}=\nabla \cdot\left(\nabla V p_{t}\right)+\varepsilon \Delta p_{t}
$$




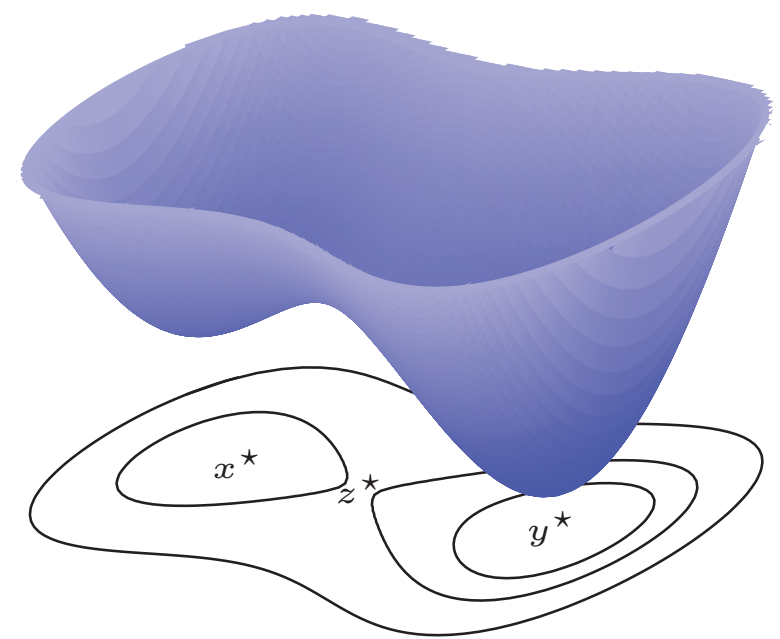

Figure 1. A double-well potential. The local minima $x^{\star}$ and $y^{\star}$ are separated by a saddle point at $z^{\star}$.

(where the operators $\nabla$ and $\Delta$ act on the variable $y$ ). The term $\nabla \cdot\left(\nabla V p_{t}\right)$ transports $p_{t}$ by a distance proportional to $-\nabla V$, while $\varepsilon \Delta p_{t}$ is a diffusion term that tends to spread the distribution of $x_{t}$. In the case of the Ornstein-Uhlenbeck process (2) one can check that

$$
p_{t}(x, y)=\frac{1}{\sqrt{2 \pi \varepsilon\left(1-\mathrm{e}^{-2 t}\right)}} \exp \left\{-\frac{\left(y-x \mathrm{e}^{-t}\right)^{2}}{2 \varepsilon\left(1-\mathrm{e}^{-2 t}\right)}\right\},
$$

that is, $x_{t}$ follows a normal law with expectation $x \mathrm{e}^{-t}$ and variance $\varepsilon\left(1-\mathrm{e}^{-2 t}\right)$. Observe that when $t$ tends to infinity this law converges to a centred normal law with variance $\varepsilon$ : the smaller the temperature, the smaller the variance and the less important the fluctuations of $x_{t}$.

For general potentials $V$, one does not know how to solve the Fokker-Planck equation (3). However, it is known that the limit as $t \rightarrow \infty$ of $p_{t}(x, y)$ is always equal to

$$
\pi(y)=\frac{1}{Z} \mathrm{e}^{-V(y) / \varepsilon},
$$

where $Z$ is a normalising constant such that the integral of $\pi(y)$ is equal to 1 . In fact, ${ }^{2} \pi(y) \mathrm{d} y$ is also an invariant probability measure of the process, that is,

$$
\int_{\mathbb{R}^{n}} \pi(x) p_{t}(x, y) \mathrm{d} x=\pi(y) \quad \forall y \in \mathbb{R}^{n}, \forall t>0 .
$$

Furthermore, one can prove that the diffusion $\left(x_{t}\right)_{t \geqslant 0}$ is $r e$ versible with respect to $\pi$ : its transition probabilities satisfy the detailed balance condition

$$
\pi(x) p_{t}(x, y)=\pi(y) p_{t}(y, x) \quad \forall x, y \in \mathbb{R}^{n}, \forall t>0 .
$$

This condition is easy to verify for the transition probabilities (4) of the Ornstein-Uhlenbeck process. From a physical point of view, it means that if we reverse the direction of time, the trajectories keep the same probabilities. Or, to put it another way, if we were to film the system, and then play the film backwards, we would be unable to tell the difference.

Metastability manifests itself in system (1) when $V$ has more than one local minimum. Let us consider the simplest

\footnotetext{
2 The invariance of $\pi$ follows from the fact that $\pi$ is in the kernel of the Fokker-Planck operator on the right-hand side of equation (3), which is equivalent to the condition $\varepsilon \nabla \cdot\left(\mathrm{e}^{-V / \varepsilon} \nabla\left(\mathrm{e}^{V / \varepsilon} \pi\right)\right)=0$. The detailed balance condition (5) follows from the fact that this operator is self-adjoint in $L^{2}$ with weight $\mathrm{e}^{V / \varepsilon}$.
}

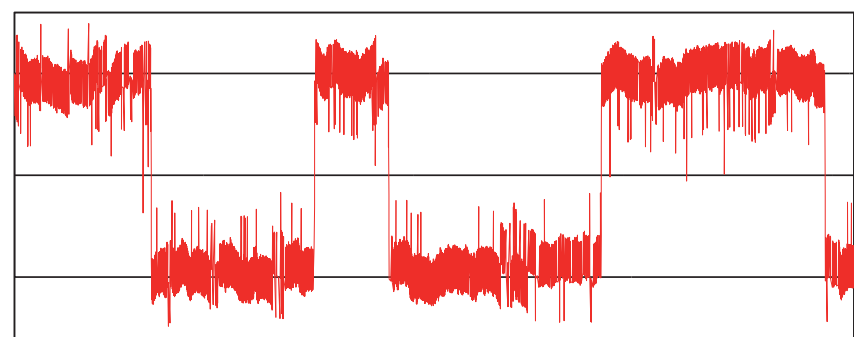

Figure 2. A trajectory $x_{t}$ of the SDE (1) in dimension 1 and for the potential $V(x)=\frac{1}{4} x^{4}-\frac{1}{2} x^{2}$. For most of the time, the trajectory keeps fluctuating around the two local minima of $V, x^{\star}=-1$ and $y^{\star}=1$, with occasional transitions from one minimum to the other. In this simulation, a relatively large $\varepsilon$ was chosen to make transitions observable during the extent of the simulation.

case when $V$ is a double-well potential, meaning that $V$ has exactly two local minima at $x^{\star}$ and $y^{\star}$, as well a saddle point at $z^{\star}$ (Figure 1). The two local minima represent two metastable states of the system, since the solutions of the SDE (1) remain in the neighbourhood of these points (Figure 2) for a long time.

The central question is then the following: let us suppose the diffusion starts in the first local minimum $x^{\star}$, and let $\mathscr{B}_{\delta}\left(y^{\star}\right)$ be a ball with small radius $\delta$ centered in the second minimum $y^{\star}$. For small $\varepsilon$, what is the behaviour of the first time $\tau=\inf \left\{t>0 \mid x_{t} \in \mathscr{B}_{\delta}\left(y^{\star}\right)\right\}$ for which $x_{t}$ visits $\mathscr{B}_{\delta}\left(y^{\star}\right)$ ?

\section{Arrhenius' Law and large-deviation theory}

A first answer to this question was given at the end of the 19th century by Jacobus van t'Hoff, then justified from a physical viewpoint by Svante Arrhenius [1]: the mean value of $\tau$ (its mathematical expectation) behaves like $\mathrm{e}^{\left[V\left(z^{\star}\right)-V\left(x^{\star}\right)\right] / \varepsilon}$. Thus, it is exponentially large in the height of the potential barrier between the two local minima of $V$. When $\varepsilon$ tends to 0 , the mean transition time tends very quickly to infinity, reflecting the fact that no transition is possible in the absence of thermal fluctuations. Conversely, when $\varepsilon$ increases, the mean transition time becomes shorter and shorter.

A rigorous version of this so-called Arrhenius law can be deduced from the theory of large deviations, developed in the SDE context by Mark Freidlin and Alexander Wentzell in the years 1960-1970 [11]. The idea is the following: fix a time interval $[0, T]$ and associate to every deterministic differentiable trajectory $\gamma:[0, T] \rightarrow \mathbb{R}^{n}$ the rate function

$$
\mathscr{I}_{[0, T]}(\gamma)=\frac{1}{2} \int_{0}^{T}\left\|\frac{\mathrm{d} \gamma}{\mathrm{d} t}(t)+\nabla V(\gamma(t))\right\|^{2} \mathrm{~d} t .
$$

Observe that this function vanishes if and only if $\gamma(t)$ satisfies $\frac{\mathrm{d} \gamma}{\mathrm{d} t}(t)=-\nabla V(\gamma(t))$, which is Equation (1) with $\varepsilon=$ 0 . Otherwise, $\mathscr{I}_{[0, T]}(\gamma)$ is strictly positive and measures the "cost" of keeping $x_{t}$ close to $\gamma(t)$. Indeed, the large-deviation principle for diffusions states that the probability that this happens is close (in a precise sense) to the exponential of $-\mathscr{I}_{[0, T]}(\gamma) /(2 \varepsilon)$

One can also estimate the probability $p(T)=\mathbb{P}^{\star}\{\tau \leqslant T\}$ of the diffusion starting from $x^{\star}$ to reach the ball $\mathscr{B}_{\delta}\left(y^{\star}\right)$ in time $T$ at most. For that, observe that, for all $T_{1} \in[0, T]$, the rate function is bounded below by $\mathscr{I}_{\left[0, T_{1}\right]}(\gamma)$, which can also 
be written as

$$
\begin{aligned}
\mathscr{I}_{\left[0, T_{1}\right]}(\gamma)=\frac{1}{2} \int_{0}^{T_{1}} \| \frac{\mathrm{d} \gamma}{\mathrm{d} t}(t) & -\nabla V(\gamma(t)) \|^{2} \mathrm{~d} t \\
& +2 \int_{0}^{T_{1}} \frac{\mathrm{d} \gamma}{\mathrm{d} t}(t) \cdot \nabla V(\gamma(t)) \mathrm{d} t .
\end{aligned}
$$

The second term on the right-hand side can be integrated and is equal to $2\left[V\left(\gamma\left(T_{1}\right)\right)-V(\gamma(0))\right]$. Since the potential along any trajectory $\gamma$ connecting $x^{\star}$ to $\mathscr{B}_{\delta}\left(y^{\star}\right)$ reaches at least the value $V\left(z^{\star}\right)$, the large-deviation principle shows that $p(T)$ is at most of order $\mathrm{e}^{-\left[V\left(z^{\star}\right)-V\left(x^{\star}\right)\right] / \varepsilon}$. Furthermore, one can construct a trajectory from $x^{\star}$ to $\mathscr{B}_{\delta}\left(y^{\star}\right)$ with cost $2\left[V\left(z^{\star}\right)-V\left(x^{\star}\right)+R(T)\right]$, where $R(T)$ is a term converging to 0 as $T \rightarrow \infty .{ }^{3}$ The argument is concluded by comparing the system with a Bernoulli process performing independent attempts of reaching $\mathscr{B}_{\delta}\left(y^{\star}\right)$ in time intervals $[k T,(k+1) T]$, each with probability of success equal to $p(T)$, whose expectation is equal $1 / p(T)$. The errors made in comparing the two processes become negligible when $\varepsilon \rightarrow 0 .^{4}$

\section{The Eyring-Kramers Law and potential theory}

The Eyring-Kramers law, proposed in the 1930s [9, 14], is more precise than Arrhenius' law ${ }^{5}$, since it describes the prefactor of the mean transition time. Let us denote by Hess $V(x)$ the Hessian matrix of the potential $V$ at $x$, which will always be assumed to be non-singular (i.e., with nonzero determinant). All eigenvalues of the matrix Hess $V\left(x^{\star}\right)$ are positive, whereas Hess $V\left(z^{\star}\right)$ has a single negative eigenvalue that will be denoted by $\lambda_{-}\left(z^{\star}\right){ }^{6}$

In this case, the Eyring-Kramers law states that

$\mathbb{E}^{x^{\star}}[\tau]=\frac{2 \pi}{\left|\lambda_{-}\left(z^{\star}\right)\right|} \sqrt{\frac{\left|\operatorname{det} \operatorname{Hess} V\left(z^{\star}\right)\right|}{\operatorname{det} \operatorname{Hess} V\left(x^{\star}\right)}} \mathrm{e}^{\left[V\left(z^{\star}\right)-V\left(x^{\star}\right)\right] / \varepsilon}[1+R(\varepsilon)]$,

where $R(\varepsilon)$ is a remainder converging to zero when $\varepsilon \rightarrow 0$. There are at present several methods to prove this result. In the following we will explain the method based on potential theory, developed by Anton Bovier, Michael Eckhoff, Véronique Gayrard and Markus Klein in the early 2000s [6], which is generalisable to the case of stochastic PDEs (readers who are not interested in these technical details are invited to go directly to section 3 ).

Let us fix two disjoint sets, $A, B \subset \mathbb{R}^{n}$, with smooth boundaries - think of neighbourhoods of the minima $x^{\star}$ and $y^{\star}$ of the potential $V$. The basic observation is that Dynkin's

For $T$ sufficiently large, one connects points close to $x^{\star}$ and $z^{\star}$ in a time $(T-1) / 2$ by a trajectory on which $\frac{\mathrm{d} \gamma}{\mathrm{d} t}(t)=+\nabla V(\gamma(t))$, with cost close to $2\left[V\left(z^{\star}\right)-V\left(x^{\star}\right)\right]$. Then, one connects a point close to $z^{\star}$ to $\mathscr{B}_{\delta}\left(y^{\star}\right)$ in a time $(T-1) / 2$ by a zero-cost deterministic trajectory. Finally, one uses the remaining time 1 to connect these pieces of trajectory with straight line segments of negligible cost.

4 The precise statement of this result is that the mean transition time $\mathbb{E}^{x^{\star}}[\tau]$ satisfies $\lim _{\varepsilon \rightarrow 0} \varepsilon \log \mathbb{E}^{x^{\star}}[\tau]=V\left(z^{\star}\right)-V\left(x^{\star}\right)$.

5 The Eyring-Kramers law was indeed proposed about three decades before the first proof of Arrhenius' law.

6 Indeed, if Hess $V\left(z^{\star}\right)$ had more negative eigenvalues, one would be able to find a more economical path, in terms of maximal height, to go from $x^{\star}$ to $y^{\star}$. For example, in dimension 2, the stationary points of $V$ at which the Hessian has two negative eigenvalues are local maxima of $V$, whereas we are interested in saddle points characterised by one positive and one negative eigenvalues.

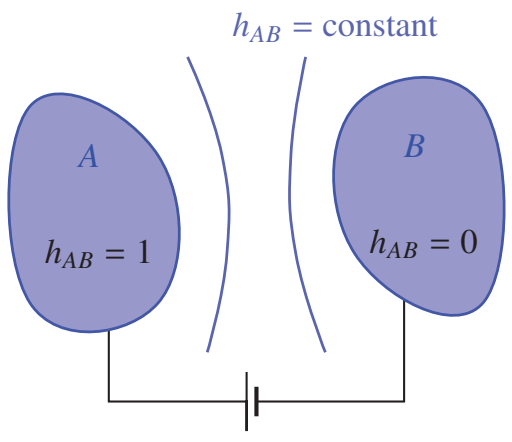

Figure 3. In the case $V=0$, the equilibrium potential $h_{A B}$ describes the electric potential of a capacitor formed by two conductors $A$ and $B$ at potentials 1 and 0 , respectively.

formula (or Itô's formula for stopping times) allows us to express several probabilistic quantities of interest as solutions of partial differential equations. For example, the function $w_{B}(x)=\mathbb{E}^{x}\left[\tau_{B}\right]$, giving the expected value of the time to reach $B$ starting from $x$, satisfies the Poisson problem

$$
\begin{cases}\left(\mathscr{L} w_{B}\right)(x)=-1 & x \in B^{c}, \\ w_{B}(x)=0 & x \in B,\end{cases}
$$

where $\mathscr{L}$ is the differential operator

$$
\mathscr{L}=\varepsilon \Delta-\nabla V \cdot \nabla,
$$

called the generator of the diffusion $\left(x_{t}\right)_{t \geqslant 0}$ (it is the adjoint in $L^{2}$ of the Fokker-Planck operator appearing in (3)).

The solution of the Poisson equation (8) can be represented in the form

$$
w_{B}(x)=-\int_{B^{c}} G_{B^{c}}(x, y) \mathrm{d} y,
$$

where $G_{B^{c}}$ is the Green function associated to $B^{c}$, solution of

$$
\begin{cases}\left(\mathscr{L} G_{B^{c}}\right)(x, y)=\delta(x-y) & x \in B^{c}, \\ G_{B^{c}}(x, y)=0 & x \in B .\end{cases}
$$

Reversibility implies that $G_{B^{c}}$ satisfies the detailed balance relation

$$
\mathrm{e}^{-V(x) / \varepsilon} G_{B^{c}}(x, y)=\mathrm{e}^{-V(y) / \varepsilon} G_{B^{c}}(y, x) \quad \forall x, y \in B^{c} .
$$

In the case $V=0$, Green's function has an electrostatic interpretation: $G_{B^{c}}(x, y)$ is the value at $x$ of the electric potential generated by a unit electric charge at $y$ when the region $B$ is occupied by a conductor at zero potential.

A second important quantity is the equilibrium potential $h_{A B}(x)=\mathbb{P}^{x}\left\{\tau_{A}<\tau_{B}\right\}$, also called committor: it gives the probability, starting from $x$, of reaching the set $A$ before reaching $B$. It is an $\mathscr{L}$-harmonic function that satisfies the Dirichlet problem

$$
\begin{cases}\left(\mathscr{L} h_{A B}\right)(x)=0 & x \in(A \cup B)^{c}, \\ h_{A B}(x)=1 & x \in A, \\ h_{A B}(x)=0 & x \in B .\end{cases}
$$

The equilibrium potential also admits an integral expression in terms of Green's function, namely

$$
h_{A B}(x)=-\int_{\partial A} G_{B^{c}}(x, y) e_{A B}(\mathrm{~d} y),
$$

where $e_{A B}$ is a measure concentrated in $\partial A$, called equilibrium measure, defined by

$$
e_{A B}(\mathrm{~d} x)=\left(-\mathscr{L} h_{A B}\right)(\mathrm{d} x) .
$$




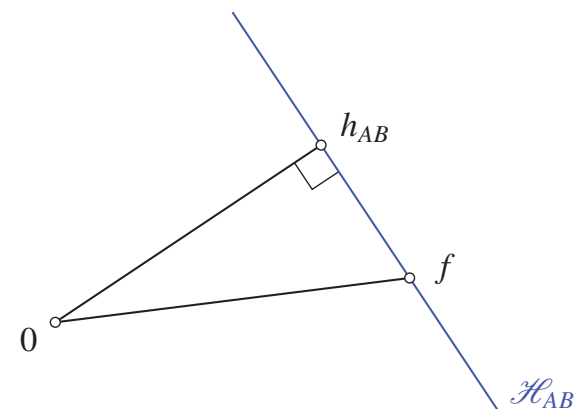

Figure 4. The Dirichlet principle states that the capacity minimises the distance to the origin, measured by the Dirichlet form, in the set $\mathscr{H}_{A B}$ of functions $h$ with value 1 in $A$ and 0 in $B$. This is due to the fact that $\left\langle f,-\mathscr{L} h_{A B}\right\rangle_{\pi}$ is constant for $f \in \mathscr{H}_{A B}$.

The electrostatic interpretation of $h_{A B}$ is that it is the electric potential of a capacitor made of two conductors in $A$ and $B$ at potentials 1 and 0, respectively (Figure 3). Finally, the capacity

$$
\operatorname{cap}(A, B)=\int_{\partial A} \mathrm{e}^{-V(x) / \varepsilon} e_{A B}(\mathrm{~d} x)
$$

is the normalising constant ensuring that

$$
v_{A B}(\mathrm{~d} x)=\frac{1}{\operatorname{cap}(A, B)} \mathrm{e}^{-V(x) / \varepsilon} e_{A B}(\mathrm{~d} x)
$$

is a probability measure on $\partial A$. In electrostatics, $\operatorname{cap}(A, B)$ is the total charge in the capacitor (which is equal to its capacity for a unit potential difference).

Combining the expressions (9) of $w_{B}$ and (11) of $h_{A B}$ with the detailed balance relation (10) of the Green function, we obtain

$$
\int_{\partial A} \mathbb{E}^{x}\left[\tau_{B}\right] v_{A B}(\mathrm{~d} x)=\frac{1}{\operatorname{cap}(A, B)} \int_{B^{c}} \mathrm{e}^{-V(x) / \varepsilon} h_{A B}(x) \mathrm{d} x,
$$

which is a fundamental relation for the potential theoretic approach. Indeed, taking for $A$ a small ball centred at $x^{\star}$, one can prove (either using Harnack inequalities, or by a coupling argument) that $\mathbb{E}^{x}\left[\tau_{B}\right]$ changes very little in $\partial A$. The left-hand side of (12) is, thus, close to the expected value $\mathbb{E}^{x^{\star}}\left[\tau_{B}\right]$. As for the right-hand side, we start by observing that if $B$ is a small ball centred in $y^{\star}$, then $h_{A B}$ is close to 1 in the basin of attraction of $y^{\star}$. Thus, Laplace's method allows us to show that

$$
\int_{B^{c}} \mathrm{e}^{-V(x) / \varepsilon} h_{A B}(x) \mathrm{d} x \simeq \sqrt{\frac{(2 \pi \varepsilon)^{n}}{\operatorname{det} \operatorname{Hess} V\left(x^{\star}\right)}} \mathrm{e}^{-V\left(x^{\star}\right) / \varepsilon} .
$$

It remains to estimate the capacity, which can be done with the help of variational principles. The Dirichlet form is the quadratic form associated to the generator, and can be written using an integration by parts (Green's identity) as

$$
\mathscr{E}(f, f)=\langle f,-\mathscr{L} f\rangle_{\pi}=\varepsilon \int_{\mathbb{R}^{n}} \mathrm{e}^{-V(x) / \varepsilon}\|\nabla f(x)\|^{2} \mathrm{~d} x,
$$

where $\langle f, g\rangle_{\pi}$ is the inner product with weight $\pi(x)$. The Dirichlet principle states that the capacity $\operatorname{cap}(A, B)$ is equal to the infimum of the Dirichlet form over all functions with value 1 in $A$, and 0 in $B$, and this infimum is attained at $f=h_{A B}$. This is a direct consequence of the fact that $\left\langle f,-\mathscr{L} h_{A B}\right\rangle_{\pi}=\operatorname{cap}(A, B)$ for all $f$ satisfying these same boundary conditions, and the Cauchy-Schwarz inequality (see Figure 4). In electrostatics, the Dirichlet form is interpreted as the electrostatic energy of the capacitor, which is indeed minimal at the equilibrium state.

A lower bound for the capacity can be obtained with the help of Thomson's principle. Given a vector field $\varphi: \mathbb{R}^{n} \rightarrow$ $\mathbb{R}^{n}$, we define the quadratic form

$$
\mathscr{D}(\varphi, \varphi)=\frac{1}{\varepsilon} \int_{(A \cup B)^{c}} \mathrm{e}^{V(x) / \varepsilon}\|\varphi(x)\|^{2} \mathrm{~d} x .
$$

Thomson's principle states that the inverse of the capacity is the infimum of $\mathscr{D}$ over all divergence-free vector fields, whose flux through $\partial A$ is equal to 1 .

Choosing appropriate test functions in both variational principles (which can be guessed from the explicitly solvable one-dimensional case), one obtains

$$
\operatorname{cap}(A, B) \simeq \frac{\left|\lambda_{-}\left(z^{\star}\right)\right|}{2 \pi} \sqrt{\frac{(2 \pi \varepsilon)^{n}}{\left|\operatorname{det} \operatorname{Hess} V\left(z^{\star}\right)\right|}} \mathrm{e}^{-V\left(z^{\star}\right) / \varepsilon} .
$$

Combining (13) and this last expression yields the EyringKramers formula (7).

\section{Metastability in the Allen-Cahn equation}

We would now like to quantify, in a way similar to what was just done in the context of SDEs, the phenomenon of metastability in stochastic partial differential equations (SPDEs). We shall consider the Allen-Cahn equation

$$
\partial_{t} \phi=\Delta \phi+\phi-\phi^{3}+\sqrt{2 \varepsilon} \xi,
$$

which is a simple model for phase separation, for instance in a mixture of ice and liquid water, or in an alloy. It is also one of the simplest SPDEs displaying metastable behaviour.

The unknown $\phi(t, x)$ is a scalar field, where the space variable $x$ lies in the torus $\mathbb{T}_{L}^{d}=(\mathbb{R} / L \mathbb{Z})^{d}$ of size $L$ (we could work on the unit torus provided we introduce a viscosity parameter multiplying the Laplacian). The term $\xi$ denotes a so-called space-time white noise. Intuitively, $\xi$ represents a Brownian noise acting independently in each point of space, meaning that

$$
\mathbb{E}[\xi(t, x) \xi(s, y)]=\delta(t-s) \delta(x-y) .
$$

Mathematically, $\xi$ is a centred Gaussian random distribution with covariance

$$
\mathbb{E}\left[\left\langle\xi, \varphi_{1}\right\rangle\left\langle\xi, \varphi_{2}\right\rangle\right]=\left\langle\varphi_{1}, \varphi_{2}\right\rangle_{L^{2}}
$$

for all pairs of test functions $\varphi_{1}, \varphi_{2} \in L^{2}$. Indeed, formally replacing the test functions by Dirac distributions we recover relation (15). Furthermore, if $\varphi_{T}(t, x)=1$ when $t \in[0, T]$ and $x$ belongs to a set $A \subset \mathbb{T}_{L}^{d}$, and is otherwise equal to 0 , then $W_{T}=\left\langle\xi, \varphi_{T}\right\rangle$ is a Brownian motion.

We can consider (14) as an infinite-dimensional analogue of the gradient diffusion (1) for the potential

$$
V(\phi)=\int_{\mathbb{T}_{L}^{d}}\left(\frac{1}{2}\|\nabla \phi(x)\|^{2}-\frac{1}{2} \phi(x)^{2}+\frac{1}{4} \phi(x)^{4}\right) \mathrm{d} x .
$$

Indeed, for all periodic functions $\psi$, the Gâteaux derivative of $V$ in the direction $\psi$ is

$$
\begin{aligned}
& \lim _{h \rightarrow 0} \frac{V(\phi+h \psi)-V(\phi)}{h} \\
&=\int_{\mathbb{T}_{L}^{d}}\left(\nabla \phi(x) \cdot \nabla \psi(x)-\phi(x) \psi(x)+\phi(x)^{3} \psi(x)\right) \mathrm{d} x,
\end{aligned}
$$


and an integration by parts of the term $\nabla \phi \cdot \nabla \psi$ shows that this derivative is equal to $-\left\langle\Delta \phi+\phi-\phi^{3}, \psi\right\rangle_{L^{2}}$ which is, up to a change of sign, exactly the inner product of the right-hand side of (14) with $\psi$.

In the deterministic case $\varepsilon=0$, the stationary solutions of (14) are the critical points of $V$, among which only two are local minima, and thus play the same role as $x^{\star}$ and $y^{\star}$ in the case of diffusions: these are the solutions identically equal to \pm 1 that we shall denote by $\phi_{ \pm}^{\star}$. If $\phi$ represents a mixture of ice and water, then $\phi_{-}^{\star}$ and $\phi_{+}^{\star}$ represent pure ice and pure water, respectively. Depending on the size $L$ of the spatial domain, there can be additional critical points. In what follows, in order to simplify the presentation, we shall concentrate our attention on the case $L<2 \pi$. In this case there is only one other critical point: the identically zero function that we shall denote by $\phi_{\text {trans }}^{\star}$, since it represents the transition state when going from $\phi_{-}^{\star}$ to $\phi_{+}^{\star}$. It plays the same role as $z^{\star}$ in the case of diffusions.

Figure 5 illustrates the time evolution of a solution of (14) in dimension $2 .^{7}$ It presents a phenomenon of gradual phase separation (in solid state physics, for example in phase separation in an alloy, one speaks of spinodal decomposition), and corresponds to a rather slow convergence towards one of the equilibria $\phi_{ \pm}^{\star}$. A difference with the one-dimensional case represented in Figure 2 is that here, one observes the coexistence of the two phases for a long time. Only after an extended time period (exceeding what is shown in Figure 5) does the system approach a single pure phase, be it blue or red. This is due to the fact that the initial condition, which is random and has zero mean, causes the field to first approach the saddle point $\phi_{\text {trans }}^{\star}$ (that also has zero mean) before being attracted by $\phi_{-}^{\star}$ or $\phi_{+}^{\star}$. Moreover, since we are in an infinite-dimensional situation, the system has plenty of "space" to evolve in before converging towards an equilibrium.

If, unlike what is shown in Figure 5, we were to start the simulation in one of the pure phases, say in $\phi_{-}^{\star}$, we would see the system stay close to that state for a very long time, before making a transition to the other state $\phi_{+}^{\star}$. Then, after another very long time period has ellapsed, we would see the system return to the initial state, and so on. The mean value of the field would then behave as illustrated in Figure 2. Therefore, we are indeed dealing with a metastability phenomenon. A natural question that arises when $\varepsilon>0$ is the following: if we start with an initial condition close to $\phi_{-}^{\star}$, what is the precise asymptotics of the time needed to reach a small neighbourhood (in an appropriate norm) of the solution $\phi_{+}^{\star}$ ?

Dimension 1: Fredholm determinants

In the case of dimension $d=1$, William Faris and Giovanni Jona-Lasinio proved in [10] a large-deviation principle with rate function (compare with the expression (6) for the rate

Animations can be found on the web pages www.idpoisson.fr/berglund/ simchain.html for dimension 1, and www.idpoisson.fr/berglund/simac. html for dimension 2. See also the YouTube page tinyurl.com/q43b6lf. Furthermore, one can find interactive simulations at the addresses experiences.math.cnrs.fr/Equation-aux-Derivees-Partielles.html and experiences.math.cnrs.fr/EQuation-aux-Derivees-Partielles-69.html.
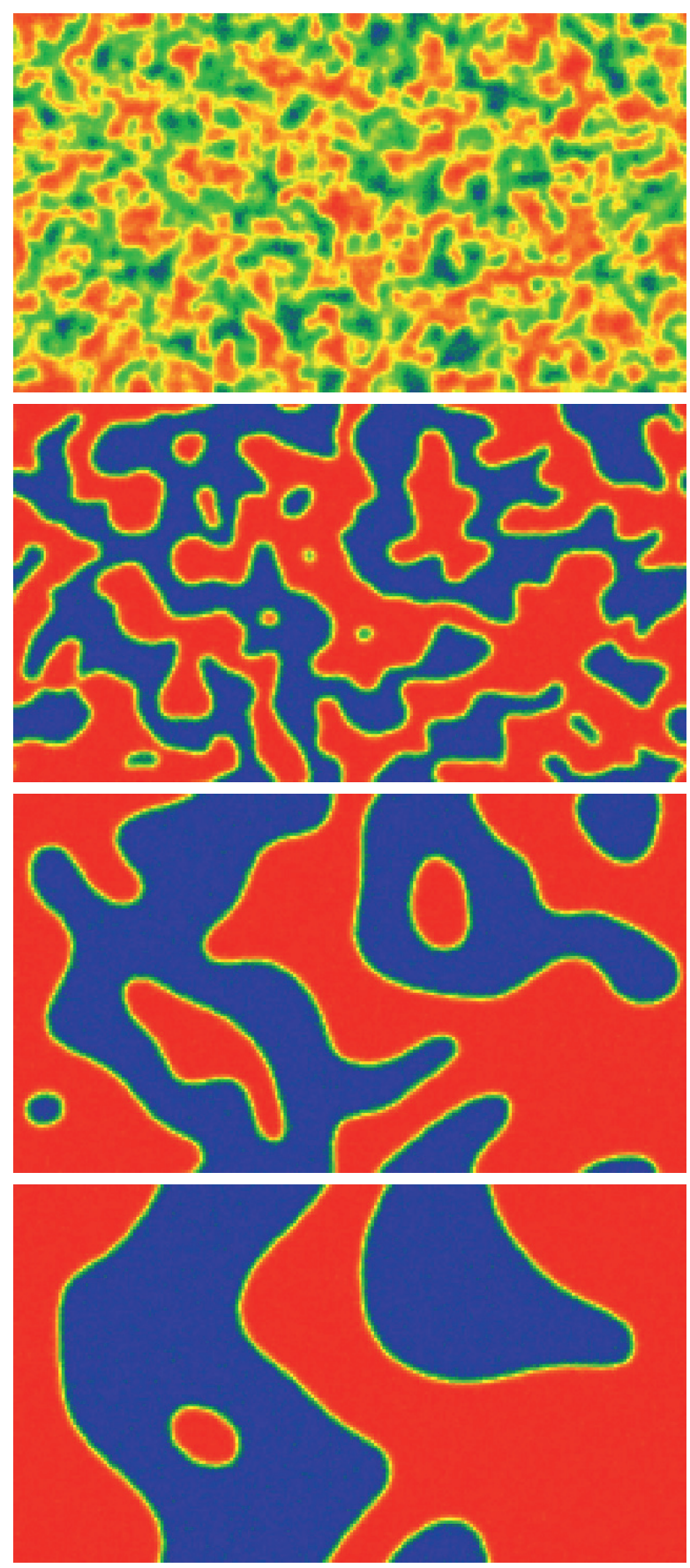

Figure 5. Time evolution of a solution of the stochastic Allen-Cahn equation on a two-dimensional torus, illustrating the phenomenon of spinodal decomposition, or slow phase separation. Red and blue colours represent regions where the field $\phi$ is close to 1 and to -1 , respectively, whereas yellow corresponds to $\phi$ close to 0 . The main effect of the noise in these simulations is to make the regions of red and blue phases slightly granular. The interfaces between the two phases remain relatively smooth due to the regularising effect of the Laplacian.

function of a diffusion)

$$
\begin{aligned}
\mathscr{I}_{[0, T]}(\gamma)=\frac{1}{2} \int_{0}^{T} \int_{\mathbb{T}_{L}}\left[\frac{\partial \gamma}{\partial t}(t, x)-\frac{\partial^{2} \gamma}{\partial x^{2}}(t, x)\right. & \\
& \left.-\gamma(t, x)+\gamma(t, x)^{3}\right]^{2} \mathrm{~d} x \mathrm{~d} t .
\end{aligned}
$$

Let $\tau$ be the first-hitting time of a ball $B=\left\{\phi:\left\|\phi-\phi_{+}^{\star}\right\|_{L^{\infty}}<\delta\right\}$, with small radius $\delta>0$ independent of $\varepsilon$. By a method similar to the one discussed in section 2, one obtains that $\tau$ satisfies 
Arrhenius' law

$$
\mathbb{E}^{\phi_{-}^{\star}}[\tau] \simeq \mathrm{e}^{\left[V\left(\phi_{\text {trans }}^{\star}\right)-V\left(\phi_{-}^{\star}\right)\right] / \varepsilon} .
$$

What about the Eyring-Kramers law? If we want to extend Expression (7), valid in finite dimension, to the present situation, we first need to determine what the analogues of the Hessian matrices of $V$ at the critical points are. An integration by parts shows that the expansion up to order 2 of the potential around $\phi_{\text {trans }}^{\star}=0$ is

$$
V(\phi)=\frac{1}{2}\langle\phi,[-\Delta-1] \phi\rangle_{L^{2}}+O\left(\phi^{4}\right),
$$

and thus we can identify Hess $V\left(\phi_{\text {trans }}^{\star}\right)$ with the quadratic form $-\Delta-1$. A similar argument applied to $\phi_{-}^{\star}$ shows that Hess $V\left(\phi_{-}^{\star}\right)$ can be identified with $-\Delta+2 .{ }^{8}$ Taken separately, these two operators do not have a well-defined determinant. However, we can write their ratio as

$$
\operatorname{det}\left((-\Delta+2)(-\Delta-1)^{-1}\right)=\operatorname{det}\left(11+3(-\Delta-1)^{-1}\right) \text {. }
$$

This is a Fredholm determinant, an object generalising the characteristic polynomial of a matrix to infinite-dimensional operators $^{9}$. To see that this determinant converges, let us observe that the eigenvalues $\lambda_{k}$ of $3(-\Delta-1)^{-1}$ decrease like $1 / k^{2}$ for large $k$. Hence, the logarithm of the determinant behaves like the sum of $\log \left(1+\lambda_{k}\right)$, that is, like the sum of the $\lambda_{k}$, i.e., the trace of $3(-\Delta-1)^{-1}$. By Riemann's criterion this sum converges, and one says that $3(-\Delta-1)^{-1}$ is trace class. In fact, using two of Euler's identities about infinite products, one can obtain the explicit value

$$
\operatorname{det}\left(11+3(-\Delta-1)^{-1}\right)=-\frac{\sinh ^{2}(L / \sqrt{2})}{\sin ^{2}(L / 2)} .
$$

The following theorem is a particular case of a result proved in [5] (and also of a result in [2] obtained by a different approach.)

Theorem 3.1. For $L<2 \pi$, one has

$$
\mathbb{E}^{\phi_{-}^{\star}}[\tau]=\frac{2 \pi}{\left|\lambda_{-}\left(\phi_{\text {trans }}^{\star}\right)\right|} \frac{\mathrm{e}^{\left[V\left(\phi_{\text {trans }}^{\star}\right)-V\left(\phi_{-}^{*}\right)\right] / \varepsilon}}{\sqrt{\left|\operatorname{det}\left(11+3(-\Delta-1)^{-1}\right)\right|}}[1+R(\varepsilon, \delta)],
$$

where $\lambda_{-}\left(\phi_{\text {trans }}^{\star}\right)=-1$ is the smallest eigenvalue of $-\Delta-1$, and $R(\varepsilon, \delta)$ converges to 0 as $\varepsilon \rightarrow 0$. (The speed of this convergence depends on $L$, it becomes slower as $L$ gets closer to $2 \pi$.)

Let us give an idea of the proof of this theorem. The first step consists of a spectral Galerkin approximation. Let $\left\{e_{k}\right\}_{k \in \mathbb{Z}}$ be a Fourier basis of $L^{2}\left(\mathbb{T}_{L}\right)$, and for a positive integer $N$ (called the ultraviolet cutoff parameter), let $P_{N}$ be the projection on the space $\mathscr{H}_{N}$ generated by $\left\{e_{k}\right\}_{|k| \leqslant N}$. The projected equation

$$
\partial_{t} \phi_{N}=\Delta \phi_{N}+\phi_{N}-P_{N}\left(\phi_{N}^{3}\right)+\sqrt{2 \varepsilon} P_{N} \xi
$$

\footnotetext{
8 The values -1 et 2 are the second derivatives of the function $\phi \mapsto \frac{1}{4} \phi^{4}-$ $\frac{1}{2} \phi^{2}$ at 0 and -1 , respectively.

9 The nonzero roots of the characteristic polynomial $c_{M}(t)=\operatorname{det}(t \mathbb{1}-M)$ of a matrix $M$ are the inverses of the roots of $\bar{c}_{M}(s)=\operatorname{det}(1-s M)$. The Fredholm determinant of $-s M$ is the analogue of $\bar{c}_{M}(s)$ when $M$ is an infinite-dimensional linear operator.
}

is equivalent to a finite-dimensional SDE of type (1), with $V$ the potential (16) restricted to $\mathscr{H}_{N}$. We can then apply the potential theoretic approach discussed in section 2 above, carefully controlling the dependence of the error terms upon the cutoff parameter $N$ and then letting $N \rightarrow \infty$.

A major difficulty is thus to get an estimate similar to (19) for the Galerkin approximation, with an error term $R(\varepsilon, \delta)$ independent of $N$. A key idea of the proof consists in decomposing the potential $V$ into a quadratic part and a higher-order part. This allows for the interpretation of the capacity and of the integral on the right-hand side of Relation (12) as expectations, under a Gaussian measure, of certain random variables that can then be estimated with the help of probabilistic arguments. Details of these computations can be found in [3, Section 2.7].

\section{Dimension 2: Carleman-Fredholm determinants}

We will now consider the Allen-Cahn equation (14) on the torus of dimension $d=2$. It turns out that, unlike in the case $d=1$, the equation is no longer well posed! This is due to the fact that space-time white noise is more singular in dimension 2 than in dimension 1. In [7], Giuseppe Da Prato and Arnaud Debussche solved this problem by a renormalisation procedure inspired by Quantum Field Theory. Instead of (14) they considered, for $\delta>0$, the regularised equation

$$
\partial_{t} \phi=\Delta \phi+\phi+3 \varepsilon C_{\delta} \phi-\phi^{3}+\sqrt{2 \varepsilon} \xi^{\delta} .
$$

Here $\xi^{\delta}$ is a regularisation of space-time white noise defined as the convolution $\varrho^{\delta} * \xi$, where

$$
\varrho^{\delta}(t, x)=\frac{1}{\delta^{4}} \varrho\left(\frac{t}{\delta^{2}}, \frac{x}{\delta}\right),
$$

for a test function $\varrho$ with integral 1 . Consequently, $\varrho^{\delta}$ converges to the Dirac distribution when $\delta$ converges to 0 . Furthermore, $C_{\delta}$ is a renormalisation constant that diverges like $\log \left(\delta^{-1}\right)$ as $\delta$ tends to 0 . Since $\xi^{\delta}$ is a function, and not a distribution, the so-called renormalised equation (20) admits solutions for all values of $\delta>0$. Da Prato and Debussche then showed that these solutions converge to a well-defined limit when $\delta$ goes to 0 .

At first sight, one might think that the stable equilibrium states of equation (20) are located at $\pm \sqrt{1+3 \varepsilon C_{\delta}}$, and thus go to infinity as $\delta$ tends to 0 with $\varepsilon$ fixed. In fact, this is not the case - a first indication of this was the proof by Martin Hairer and Hendrik Weber in [13] of a large-deviation principle with rate function analogous to that of the one dimensional case (see (17)). The key observation is that, as in dimension 1, this rate function does not include any renormalisation counterterm. This implies the Arrhenius law

$$
\mathbb{E}^{\phi_{-}^{\star}}[\tau] \simeq \mathrm{e}^{\left[V\left(\phi_{\text {trans }}^{\star}\right)-V\left(\phi_{-}^{\star}\right)\right] / \varepsilon},
$$

where $V$ is the potential (16) without renormalisation term. As before, $\tau$ is the transition time between the equilibria $\phi_{-}^{\star}$ and $\phi_{+}^{\star}$, located at \pm 1 , respectively. We can interpret this result as indicating that the only role of the counterterm $3 \varepsilon C_{\delta} \phi$ is to make the nonlinearity $\phi^{3}$ well defined.

What about the Eyring-Kramers law? It turns out that the Fredholm determinant (18) does not converge. In fact, $3(-\Delta-1)^{-1}$ is no longer of trace class in dimension 2 , since its eigenvalues are proportional to $1 /\left(k_{1}^{2}+k_{2}^{2}\right)$ with $k_{1}$ and $k_{2}$ 
two nonzero integers, and hence the sum of these eigenvalues diverges like the harmonic series!

The solution to this problem consists, first of all, in working, as in dimension 1, with a spectral Galerkin approximation with ultraviolet cutoff $N$. Instead of regularising the spacetime white noise by convolution, one can again consider its spectral Galerkin projection $\xi_{N}=P_{N} \xi$, with a counterterm

$$
3 \varepsilon C_{N}=\frac{3 \varepsilon}{L^{2}} \operatorname{Tr}\left(P_{N}(-\Delta-1)^{-1}\right)
$$

that diverges as $\log (N)$ (the constant $C_{N}$ is the variance of the truncated Gaussian free field $\left.{ }^{10}\right)$. The renormalised potential can thus be written as

$$
V_{N}(\phi)=\int_{\mathbb{T}_{L}^{2}}\left[\frac{1}{2}\|\nabla \phi(x)\|^{2}+\frac{1}{4} \phi(x)^{4}-\frac{1}{2}\left(1+3 \varepsilon C_{N}\right) \phi(x)^{2}\right] \mathrm{d} x .
$$

The crucial point is to observe that

$$
V_{N}\left(\phi_{\text {trans }}^{\star}\right)-V_{N}\left(\phi_{-}^{\star}\right)=\frac{L^{2}}{4}+\frac{3}{2} L^{2} \varepsilon C_{N} .
$$

The new term $\frac{3}{2} L^{2} \varepsilon C_{N}$ is exactly the one that will make the prefactor converge. Indeed, the Eyring-Kramers formula involves the factor

$$
\operatorname{det}\left(11+3 P_{N}(-\Delta-1)^{-1}\right) \mathrm{e}^{-3 \operatorname{Tr}\left(P_{N}(-\Delta-1)^{-1}\right)},
$$

which does have a limit as $N \rightarrow \infty$ (this follows from the fact that its logarithm behaves like the sum of $\left.1 /\left(k_{1}^{2}+k_{2}^{2}\right)^{2}\right)$. This is, in fact, a known regularisation of the Fredholm determinant, also called the Carleman-Fredholm determinant, sometimes denoted $\operatorname{det}_{2}\left(1+3(-\Delta-1)^{-1}\right)$. Unlike Fredholm's determinant, this modified determinant is well defined for operators whose square is trace class, the so-called Hilbert-Schmidt operators, which include $3(-\Delta-1)^{-1}$.

The following theorem combines results of [4] and [15].

Theorem 3.2. Let $\tau$ be the first-hitting time of a ball (in the Sobolev norm $H^{s}$ for some $\left.s<0\right)$, centred at $\phi_{+}^{\star}$. For $L<2 \pi$, we have

$$
\mathbb{E}^{\phi_{-}^{\star}}[\tau]=\frac{2 \pi}{\left|\lambda_{-}\left(\phi_{\text {trans }}^{\star}\right)\right|} \frac{\mathrm{e}^{\left[V\left(\phi_{\text {trans }}^{\star}\right)-V\left(\phi_{-}^{*}\right)\right] / \varepsilon}}{\sqrt{\left|\operatorname{det}_{2}\left(11+3(-\Delta-1)^{-1}\right)\right|}}[1+R(\varepsilon, \delta)],
$$

where $\lambda_{-}\left(\phi_{\text {trans }}^{\star}\right)=-1$ is the smallest eigenvalue of $-\Delta-1$, and $R(\varepsilon, \delta)$ is an error term converging to 0 as $\varepsilon \rightarrow 0$ (at a convergence rate depending on $L$.)

This result confirms that the renormalisation procedure does not displace the stationary states, since the theorem applies to the states $\phi_{ \pm}^{\star}$ located at \pm 1 . However, the renormalisation procedure is necessary to get a finite prefactor for the transition time, since the ratio of the spectral determinants and the counterterm $\frac{3}{2} L^{2} \varepsilon C_{N}$ in the potential compensate each other exactly.

\section{$4 \quad$ Some open problems}

A natural question to ask is whether an Eyring-Kramers law exists for the Allen-Cahn equation in dimension $d=3$ (in dimension $d=4$ one does not expect the existence of nontrivial solutions to this equation). As shown by Martin Hairer

\footnotetext{
10 For more information about the Gaussian free field see the article of Rémi Rhodes in the July 2018 issue of La Gazette des Mathématiciens.
}

in the widely noted paper [12] $]^{11}$, which earned him the Fields Medal in 2014, the form of the renormalised equation is

$$
\partial_{t} \phi=\Delta \phi+\phi+\left[3 \varepsilon C_{\delta}^{(1)}-9 \varepsilon^{2} C_{\delta}^{(2)}\right] \phi-\phi^{3}+\sqrt{2 \varepsilon} \xi^{\delta},
$$

where $C_{\delta}^{(1)}$ and $C_{\delta}^{(2)}$ diverge like $\delta^{-1}$ and $\log \left(\delta^{-1}\right)$, respectively. The first counterterm comes from the same renormalisation procedure as in dimension 2 (called Wick renormalisation), and does not introduce any new difficulties. On the other hand, the second counterterm is specific to dimension 3 and is at the origin of numerous problems. In particular, contrary to what happens in the case $d=2$, the invariant measure of the AllenCahn equation is singular with respect to the Gaussian free field.

However, we can note that $(-\Delta-1)^{-1}$ is Hilbert-Schmidt in dimension 3 as well. As the second counterterm occurs with a factor $\varepsilon^{2}$, we expect that an Eyring-Kramers formula analogous to (21) is still valid. With Ajay Chandra, Giacomo Di Gesù and Hendrik Weber we managed to establish some of the estimates needed to prove that result. However, so far the lower bound on the capacity still resists our efforts.

Of course, it would be desirable to obtain Eyring-Kramers formulas not just for the Allen-Cahn equation but also for other SPDEs. An example is the Cahn-Hilliard equation describing phase separation in cases where the total volume of each phase is conserved, like in mixtures of water and oil. However, as in most mathematical models of metastable systems, these SPDEs remain based on a lattice dynamics: each lattice point is characterised by its state, but remains fixed in the same place. This is a good model for certain alloys or for ferromagnetic materials, which have a crystalline structure with different types of atoms or spins attached to each site. However, for a mixture of ice and liquid water there is no underlying lattice. One of the great challenges in the theory of metastability is to analyse models, taking into account the fact that ice crystals can move through liquid water to form larger crystals by agglomeration.

\section{A Appendix: Brownian motion}

Brownian motion is a mathematical model for the erratic movement of a particle immersed in a fluid, under the effect of collisions with the fluid's molecules. It was first observed by the naturalist Robert Brown in 1827, while studying pollen grains under a microscope.

The first mathematical descriptions of Brownian motion were proposed by the French mathematician Louis Bachelier in 1901, for applications in finance, and by Albert Einstein in 1905. Variants of their approaches were developed by Marian Smoluchowski in 1906 and by Paul Langevin in 1908. Einstein's computations allowed Jean Perrin to experimentally estimate Avogadro's number in 1909, a feat that earned him the Nobel Prize in 1926.

\footnotetext{
11 For further details of the theory introduced by Martin Hairer, called theory of regularity structures, the reader may consult the paper by François Delarue in the January 2015 issue of La Gazette des Mathématiciens and the paper of Bruned, Hairer and Zambotti published in the March 2020 issue of this Newsletter.
} 

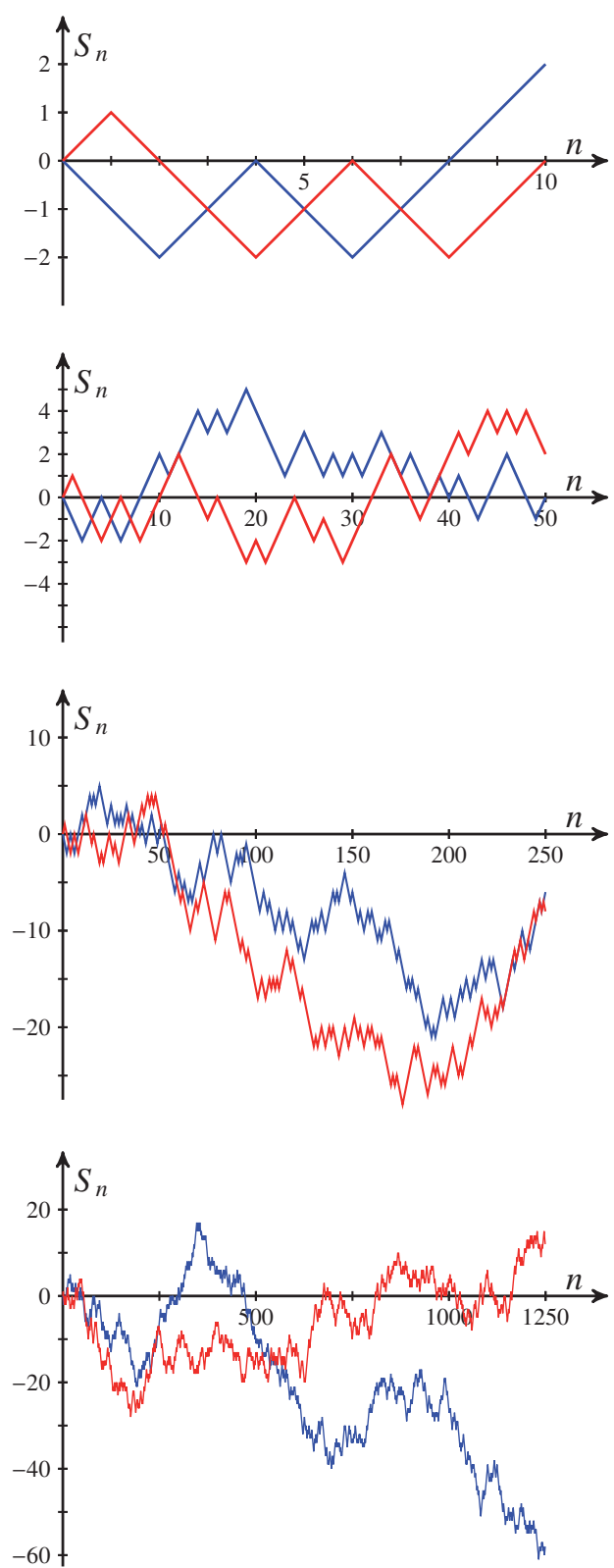

Figure 6. Two realisations (one in red and one in blue) of a symmetric random walk in $\mathbb{Z}$, seen at different scales. From one plot to the next, the horizontal scale is compressed by a factor of 5 , while the vertical scale is compressed by a factor of $\sqrt{5}$.

Let us consider the case of dimension 1 and assume that the particle undergoes regular collisions, at time intervals $\Delta t$. Between two successive collisions, the particle travels a distance $\Delta x$ with equal probability $\frac{1}{2}$ to the left or to the right. Its position at time $n \Delta t$ is thus given by $S_{n} \Delta x$, where $S_{n}$ is a sequence of integers such that the increments $S_{n+1}-S_{n}$ have value 1 or -1 , each with probability $\frac{1}{2}$. Furthermore, we assume that each increment is independent from all previous ones. The sequence $S_{n}$ is called a symmetric random walk in $\mathbb{Z}$ (see Figure 6).

Since in practice the space and time intervals $\Delta x$ and $\Delta t$ are very small, it seems relevant to let them converge to zero in order to obtain a universal object. It turns out that this limit is only interesting if $\Delta t$ is proportional $\Delta x^{2}$ (this is a conse- quence of the Central Limit Theorem). This amounts to setting

$$
W_{t}:=\lim _{n \rightarrow \infty} \frac{1}{\sqrt{n}} S_{\lfloor n t\rfloor} .
$$

This definition turns out to be equivalent to requiring that for all $t>s \geqslant 0$ the increment $W_{t}-W_{s}$ follows a centred normal law, with variance $t-s$, and independent of the values of the process up to time $s$.

Norbert Wiener showed in 1923 that the trajectories $t \mapsto$ $W_{t}$ are continuous ( $W_{t}$ is also known today as the Wiener process). Other properties of $W_{t}$ were established by several mathematicians, including Raymond Paley, Antoni Zygmund and Paul Lévy. In particular, we know that trajectories of Brownian motion are nowhere differentiable. This poses a problem for the definition of the SDE (1), which is solved by defining its solutions to be those of the integral equation

$$
x_{t}=x_{0}-\int_{0}^{t} \nabla V\left(x_{s}\right) \mathrm{d} s+\sqrt{2 \varepsilon} W_{t},
$$

that can be studied using a fixed-point argument. The theory was generalised by Kiyoshi Itô in the 1940s. His stochastic calculus allows one to solve variants of (1) in which the noise term is multiplied by a function of $x$. Some ideas at the basis of stochastic calculus were discovered independently by Wolfgang Döblin and sent to the French Academy of Sciences in a sealed envelope that was only opened in 2000 .

\section{Bibliography}

[1] S. Arrhenius. On the reaction velocity of the inversion of cane sugar by acids. J. Phys. Chem., 4:226, 1889. In German. Translated and published in: Selected Readings in Chemical Kinetics, M.H. Back and K.J. Laider (eds.), Pergamon, Oxford, 1967.

[2] F. Barret. Sharp asymptotics of metastable transition times for one dimensional SPDEs. Ann. Inst. Henri Poincaré Probab. Stat., 51(1):129-166, 2015.

[3] N. Berglund. An introduction to singular stochastic PDEs: Allen-Cahn equations, metastability and regularity structures. Lecture notes, Sarajevo Stochastic Analysis Winter School. arXiv:1901.07420, January 2019.

[4] N. Berglund, G. Di Gesù, and H. Weber. An Eyring-Kramers law for the stochastic Allen-Cahn equation in dimension two. Electron. J. Probab., 22:1-27, 2017.

[5] N. Berglund and B. Gentz. Sharp estimates for metastable lifetimes in parabolic SPDEs: Kramers' law and beyond. Electron. J. Probab., 18:no. 24, 58, 2013.

[6] A. Bovier, M. Eckhoff, V. Gayrard, and M. Klein. Metastability in reversible diffusion processes. I. Sharp asymptotics for capacities and exit times. J. Eur. Math. Soc. (JEMS), 6(4):399424, 2004.

[7] G. Da Prato and A. Debussche. Strong solutions to the stochastic quantization equations. Ann. Probab., 31(4):19001916, 2003.

[8] F. den Hollander. Metastability under stochastic dynamics. Stochastic Process. Appl., 114(1):1-26, 2004.

[9] H. Eyring. The activated complex in chemical reactions. Journal of Chemical Physics, 3:107-115, 1935.

[10] W. G. Faris and G. Jona-Lasinio. Large fluctuations for a nonlinear heat equation with noise. J. Phys. A, 15(10):3025-3055, 1982.

[11] M. I. Freidlin and A. D. Wentzell. Random Perturbations of Dynamical Systems. Springer-Verlag, New York, second edition, 1998. 
[12] M. Hairer. A theory of regularity structures. Invent. Math., 198(2):269-504, 2014.

[13] M. Hairer and H. Weber. Large deviations for white-noise driven, nonlinear stochastic PDEs in two and three dimensions. Ann. Fac. Sci. Toulouse Math. (6), 24(1):55-92, 2015.

[14] H. A. Kramers. Brownian motion in a field of force and the diffusion model of chemical reactions. Physica, 7:284-304, 1940.

[15] P. Tsatsoulis and H. Weber. Exponential loss of memory for the 2-dimensional Allen-Cahn equation with small noise. Probability Theory and Related Fields, 177:257-322, 2020. arXiv:1808.04171.

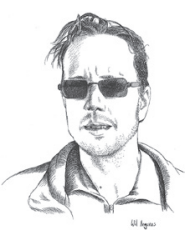

Nils Berglund obtained his PhD in mathematical physics from Ecole Polytechnique Fédérale de Lausanne (EPFL) in 1998. After doing postdocs at WIAS Berlin, Georgia Tech Atlanta and ETH Zürich, he held a full-time position at Centre de Physique Théorique in
Marseille. He is currently full professor at Institut Denis Poisson (affiliated with University of Orléans, University of Tours and CNRS). His research interests are in stochastic differential equations and stochastic partial differential equations, which are often motivated by problems in physics and biology.

Acknowledgments. This is an English translation by Fernando Pestana da Costa of the French article Métastabilité d'EDP stochastiques et déterminants de Fredholm by Nils Berglund, published in La Gazette des Mathématiciens (N. 163, January 2020). The EMS Newsletter is grateful to the author, to La Gazette des Mathématiciens, and to the Société Mathématique de France for authorisation to republish this article. The author would like to thank Damien Gayet, editor in chief of La Gazette des Mathématiciens, for his critical remarks on early French versions of this text, which greatly improved its readability. 